\title{
Atitudes e Conhecimentos de Idosos sobre Intercurso Sexual no Envelhecimento
}

\author{
Iara de França Santos ${ }^{1}$ \\ ${ }^{1}$ Universidade de Pernambuco, PE, Brasil.
}

Resumo: A sexualidade no processo de envelhecer é um fenômeno que precisa ser compreendido de forma sistematizada. Dada a amplitude de possibilidades em discussões acerca da sexualidade, o presente estudo focou no intercurso sexual no envelhecimento. Assim, o objetivo traçado foi compreender as atitudes e os conhecimentos de idosos de um município do agreste de Pernambuco sobre o intercurso sexual no envelhecimento. Foi utilizado o método quantitativo descritivo, com instrumento de coleta e de análise dos dados a escala ASKAS (Aging Sexual Knowledge and Attitudes Scale) aplicada à amostra de 150 participantes de ambos os sexos. Os resultados evidenciaram que a maioria dos entrevistados compreende que práticas sexuais podem trazer benefícios psicológicos. Apesar disso, a maior parte dos participantes, tanto das mulheres quanto dos homens, indicaram que o interesse sexual inevitavelmente desaparece depois dos 65 anos. As mulheres da amostra, como já era esperado, mostraram-se mais afetadas por tabus e proibições quanto à realização de desejos sexuais, consideraram o sexo como algo perigoso e alegaram vergonha para demonstrar interesse sexual, o que aponta para a necessidade de uma discussão de gênero.

Palavras-chave: Envelhecimento, Intercurso sexual, Atitudes.

\section{Attitudes and Knowledge of Older Adults about Sexual Intercourse in Aging}

Abstract: The sexuality in the process of aging is a phenomenon that should be understood in a systematic way. Given the number of possibilities surrounding sexuality, our study focused on sexual intercourses. Therefore, we aimed to understand the attitudes and the knowledge of the older adults from a municipality in the agreste of the state of Pernambuco about to sexual intercourse during aging. We used both quantitative and descriptive methods and the ASKAS (Aging Sexual Knowledge and Attitudes Scale) scale to collect and analyze the data of 150 participants of both sexes. The results showed that most people interviewed understand that sexual intercourses can bring some psychological benefits. Despite this, most of them pointed that sexual interest inevitably disappears after the 65 years of age. The women interviewed, as expected, were more affected by taboos and prohibition related to sexual desires, considering sex as a danger and being ashamed of showing sexual interest for their partner, pointing to the need of an open discussion on the topic.

Keywords: Aging, Sexual intercourses, Attitudes. 


\title{
Actitudes y Conocimientos de Ancianos sobre Relaciones Sexuales en el Envejecimiento
}

\begin{abstract}
Resumen: La sexualidad en el proceso de envejecimiento es un fenómeno que necesita ser entendido sistemáticamente. De la amplitud de posibilidades que permite la discusión sobre la sexualidad, el presente estudio se centró en las prácticas sexuales. Su objetivo fue comprender las actitudes y el conocimiento que los ancianos de un municipio de agreste de Pernambuco (Brasil) tenían sobre las relaciones sexuales durante el envejecimiento. Se utilizó el método descriptivo cuantitativo, con un instrumento de recopilación y análisis de datos con la escala ASKAS (Aging Sexual Knowledge and Attitudes Scale) aplicada a la muestra de 150 participantes de ambos sexos. Los resultados mostraron que la mayoría de los entrevistados entienden que las prácticas sexuales pueden traer beneficios psicológicos. Sin embargo, la mayoría de los participantes, tanto mujeres como hombres, indicaron que el interés sexual desaparece inevitablemente después de los 65 años. Como se esperaba, las mujeres de la muestra se vieron más afectadas por los tabúes y las prohibiciones sobre el cumplimiento de los deseos sexuales, consideraron que el sexo era peligroso y alegaron vergüenza para demostrar interés sexual, lo que señala la necesidad de una discusión de género.
\end{abstract}

Palabras clave: Envejecimiento, Relaciones sexuales, Actitudes.

\section{Introdução}

O envelhecimento é considerado uma fase sequenciada da existência, que se inicia no nascimento e finaliza com a morte (Papaléo Netto, 2011). A forma como os idosos atuam diante da finitude tem relação com suas experiências individuais e coletivas (questões históricas e culturais); assim, sua história de vida é determinante para compreendermos como os idosos vivenciam essa dimensão humana (R. Butler \& Lewis, 1985).

Segundo a Política Nacional do Idoso (PNI), a Lei n. 8.842/1994, idoso é a pessoa maior de 60 anos. Conforme apontam Rozendo e Alves (2015), a longevidade do brasileiro está na faixa etária acima de 75 anos. Já o Instituto Brasileiro de Geografia e Estatística (IBGE, 2018) mostra que, em 2010, o percentual de idosos no Brasil era de 7,32\% da população total e traça uma estimativa de $25,49 \%$ para 2060.

Figueiredo (2016) explica que com o aumento de idosos se faz necessário o investimento em políticas públicas que promovam um envelhecimento ativo, compreendido como um envelhecer em que o indivíduo é visto de forma integral em seus aspectos psicológicos, biológicos (físicos e sexuais) e sociais.

Para Hogan (1985), sexualidade é uma experiência que envolve tanto enfoques mentais, quanto corporais - não somente genitais. Sua construção refere-se a aspectos físicos, valores individuais, experiências vividas, comportamentos, crenças, emoções e personalidade.

Sinković e Towler (2018) realizaram uma revisão de literatura com o objetivo de analisar pesquisas qualitativas sobre sexualidade e saúde sexual de idosos, sistematizando os dados de 69 estudos. As autoras encontraram como informação frequente nos estudos a naturalização dos problemas sexuais com o envelhecimento, o que parecia modular a angústia dos idosos pesquisados em relação a suas dificuldades. Elas levantam que o estigma pode fazer com que as pessoas não busquem cuidados e incluem um estudo indicando que celibatários são mais descuidados com sua saúde e bem-estar. Segundo elas, as identidades culturas e sexuais são diversas e desempenham um papel importante na saúde sexual dos idosos. Tal indicação aponta para a necessidade de investigações também locais, em especial quando as realidades são diferentes daquelas que serviram de cenário para os artigos internacionais. $\mathrm{O}$ agreste pernambucano, com sua cultura e situação socioeconômica, por exemplo, precisa de pesquisas locais para identificar características próprias e orientar políticas públicas. 
Em um estudo transcultural, Štulhofer, Hinchliff, Jurin, Hald e Træen (2018) investigaram a relação entre envelhecimento saudável e interesse sexual. Participaram 2.461 pessoas sexualmente ativas e inativas com idades entre 60 e 75 anos. Os autores concluíram que os maiores escores de envelhecimento bem-sucedido foram consistentemente relacionados à menor redução no interesse sexual e no prazer entre homens e mulheres. Apesar de o estudo ter sido realizado na Europa, vale considerar possibilidade de sua aplicação para outras populações.

Apesar da grande variabilidade de vivências com o envelhecer, os aspectos biológicos não podem ser negligenciados e precisam compor a formação de quem atua com essa população. Conforme Alencar (2013), algumas mudanças orgânicas são distintas nos homens e nas mulheres e, consequentemente, podem interferir na atividade sexual. López Sánchez e Fuertes (1989) destacam que uma das alterações fisiológicas que ocorrem nos homens é o decréscimo na produção de esperma, além da diminuição progressiva da produção de testosterona. Sobre as mudanças ocorridas nas mulheres idosas, os mesmos autores acentuam que a menopausa pode acarretar alterações como a diminuição da mucosa vaginal, com subsequente redução da lubrificação, o que causa desconforto no início da penetração e faz se precisar de mais tempo para chegar ao orgasmo.

Dessa forma, ao formular políticas de promoção de saúde é necessário incluir não apenas a população envelhecida de forma generalizada, como também considerá-la em todas as nuances de vida, incluindo a sexualidade. Os primeiros passos para traçar metas de ação em relação à maneira de abordar e atender à população considerando esse aspecto é conhecer as atitudes sobre tal faceta da vivência humana. Diante dessa demanda, o presente estudo traçou como objetivo principal compreender as atitudes e os conhecimentos de idosos de um município do agreste de Pernambuco sobre intercurso sexual no envelhecimento.

No presente estudo foi utilizado o paradigma apresentado por Jordão Netto (1997), que evita uma visão generalizante e universalista, por considerar que o envelhecimento psicológico e social de cada pessoa depende da maneira particular como ela vivenciou as diversas situações ao longo de sua vida e na sociedade.

Por essas razões, relacionadas à manutenção da saúde integral do indivíduo, ao se intervir em uma comunidade, é importante investigar suas crenças, atitudes e informações, aliando, assim, teorias com as singularidades de cada localidade. Em relação a aspectos gerais, os profissionais de saúde precisam estar informados sobre vicissitudes próprias do envelhecer. Alencar, Marques, Leal e Vieira (2014) esclarecem que com o passar dos anos o corpo adquire algumas limitações resultantes do desgaste físico e biológico, mas isso não significa que o idoso não tenha vida sexual. É preciso discutir esse preconceito e desinformação, desmistificando tais pensamentos.

Alguns idosos começam a achar que já não têm liberdade, não se permitem pensar e agir na busca de seu bem-estar, o que pode contribuir para que eles apresentem problemas de saúde. Nessas situações, a ajuda especializada pode reduzir esses problemas e sintomas negativos (Carvalho \& Sardinha, 2017). O medo aumenta com crenças equivocadas sobre os comportamentos sexuais dos idosos existentes na sociedade. O diálogo com os profissionais de saúde é fundamental para sanar dúvidas e buscar os métodos e cuidados possíveis para cada necessidade (Alencar, 2013).

Além dos aspectos fisiológicos, precisamos considerar também os fatores psicossociais na compreensão da sexualidade humana no envelhecimento. Carvalho e Sardinha (2017) destacam que alguns padrões criados sobre o desempenho perfeito no ato sexual contribuem para o desenvolvimento de ansiedade durante a relação sexual, trazendo como consequências respostas frustrantes. Não há desempenho correto, pois cada casal e/ ou indivíduo tem sua fonte de satisfação; sendo assim, não é necessário seguir um padrão, as possibilidades tornam-se diversas e podemos experimentar cada uma delas.

Alencar (2013), ao falar sobre aspectos socioculturais que interferem na sexualidade das pessoas na terceira idade, aponta que muitos idosos não realizam práticas sexuais por não terem parceiro fixo e/ou serem viúvos, fato mais comum entre as mulheres. Os homens costumam ter um comportamento diferente: procuram outras companheiras independentemente da idade. Segundo Rozendo e Alves (2015), muitas mulheres relacionam o sexo ao casamento monogâmico, enquanto a liberdade sexual dos homens lhes permite ter várias parceiras sexuais. Essas limitações culturalmente impostas levantam a importância de se discutir as questões de gênero que refletem nas ações dos idosos e fazem com que homens e mulheres acabem não obtendo a mesma liberdade sexual, o que pode acarretar prejuízos para a saúde. 
A qualidade e cumplicidade da relação conjugal e sexual são elaboradas ao longo do relacionamento do casal. Nessas situações, a satisfação sexual, os desejos e o prazer estão relacionados às experiências individuais e às vividas com o cônjuge. Quando existe uma interação afetiva e harmoniosa, os desejos podem ser renovados e aumentarem com o passar dos anos (R. Butler e Lewis, 1985).

\section{Método}

Tendo em vista a natureza do problema de pesquisa apresentado, anteriormente foi realizado um estudo quantitativo e descritivo. Na pesquisa quantitativa coleta-se e analisa-se dados numéricos sobre variáveis determinadas, a fim de identificar as realidades, os sistemas de relações e a estrutura dinâmica do que foi investigado. Esse método pode representar a força de associação ou correlação entre variáveis, a generalização e objetivação dos resultados por meio de uma mostra específica que remete a uma população escolhida para tal estudo (Esperón, 2017). Seus resultados podem servir de base para novas pesquisas e serem elementares no desenvolvimento de estratégias que solucionem questões relevantes encontradas nos resultados do estudo. Em nosso caso, compreender os conhecimentos e atitudes pode auxiliar na implementação de práticas nos mais diversos dispositivos de saúde disponíveis no município que atendem aos idosos pesquisados, além de, claro, subsidiar práticas em outras localidades cujas populações tenham características semelhantes.

Os sujeitos que participaram da pesquisa foram contatados nas Estratégias de Saúde da Família (ESF) que frequentavam, após divulgação da pesquisa nos grupos de idosos. A coleta de dados aconteceu até completarmos $10 \%$ dos usuários frequentes de cada ESF e ao final foram respondidos 150 questionários, 102 por participantes do sexo feminino e 48, do sexo masculino.

O contato era individual, em uma sala com privacidade, e os respondentes puderam expressar-se todas as vezes que alguma afirmativa lhes remetia a questões de suas vidas. Apesar de ser um questionário, o preenchimento não foi feito pelo próprio participante - a questão era lida em voz alta e cada um tinha a possibilidade de responder conforme sua opinião. A escolha desse procedimento teve relação com a pouca escolaridade dos participantes e com a possibilidade de cuidado imediato, no caso de algum desconforto. A presente pesquisa foi aprovada pelo
Comitê de Ética sob o número do parecer 2.881.205 e CAAE: 79293517.1.0000.5207 e todas as recomendações foram respeitadas. O instrumento utilizado foi a Aging Sexual Knowledge and Attitudes Scale (ASKAS), criada por White em 1982, com objetivo de avaliar o conhecimento e as atitudes em relação à sexualidade do idoso de uma maneira indireta, abordando a opinião da pessoa sobre intercursos sexuais de forma geral no processo de envelhecimento, sem direcionar-se a hábitos individuais.

O uso da escala contribuiu para aproximação com os idosos e possibilitou conhecermos mais sobre eles, pois surgiram momentos de interação e troca de conhecimento entre pesquisadoras e pesquisados. A escala nos possibilitou ter um panorama dos conhecimentos e atitudes que os idosos possuíam sobre sexualidade na terceira idade.

A versão brasileira da escala, que recebeu o título de Escala de Atitudes e Conhecimento sobre Sexualidade no Envelhecimento (ASKAS), é composta por 28 itens. A primeira parte tem 20 perguntas referentes ao conhecimento da sexualidade na terceira idade e a segunda apresenta oito itens, que são direcionados para questões relativas às atitudes sobre sexualidade na terceira idade (Viana, 2008). As questões abordam de forma sistematizada a compreensão do respondente acerca de práticas sexuais, incluindo masturbação, adoecimento, interferência do uso de medicamentos, impotência e satisfação sexual. O instrumento não traz afirmativas acerca da percepção em relação ao próprio corpo, autoestima, empoderamento ou aspectos psicossociais relacionados ao tema.

A seguir serão apresentados os resultados encontrados, apontando inicialmente o perfil socioeconômico da população pesquisada.

\section{Resultados percentuais da pesquisa}

\section{Perfil socioeconômico}

O município em que se deu a pesquisa contava, de acordo com o censo de 2010, com 12,1\% de pessoas acima de 60 anos. Em 2015, o salário médio mensal era de 1,5 salários mínimos, o que caracteriza o perfil socioeconômico da maioria da população, que depende dos dispositivos públicos para cuidar de sua saúde (IBGE, 2010).

Dos 150 idosos que responderam à ASKAS, 68,66\% eram do sexo feminino e $31,33 \%$ do sexo masculino. 
O estado civil dos participantes foi dividido em quatro categorias: solteiro(a) (8\%), casado(a) $(62,66 \%)$, separado(a) (14,66\%) e viúvo(a) (14,16\%). O nível de escolaridade ficou em cinco categorias: não alfabetizado (32,66\%), fundamental incompleto $(30,66 \%)$, fundamental completo (17,33\%), médio incompleto $(4,66 \%)$, médio completo $(6,66 \%)$ e superior completo (8\%). Então, como perfil populacional, temos uma maioria com renda mensal média de 1,5 salários mínimos e $63,62 \%$ com pouca escolaridade (somatório de alfabetizado e fundamental incompleto). Podemos supor tratar-se de um grupo de pessoas que obteve pouco acesso à educação e que, por essa razão, pode estar em situação de vulnerabilidade, sem recursos financeiros próprios para ações de promoção de saúde. Sobre a religião dos participantes tivemos quatro categorias: católica $(84,66 \%)$, evangélica $(11,33 \%)$, espírita $(0,66 \%)$ e cristã $(3,33 \%)$.

Os resultados frente ao questionário aplicado com a população idosa serão demonstrados com os percentuais para as respostas às perguntas, assim como serão relacionados com teóricos que discutem a temática.

\section{Respostas à ASKAS}

O questionário contempla três questões acerca do interesse sexual, a saber: atividade sexual na ausência de doenças; sentimento de vergonha quanto ao tema; desaparecimento do interesse com o passar dos anos. Na opinião das mulheres $(77,45 \%)$ e dos homens $(93,75 \%)$ a atividade sexual pode trazer benefícios psicológicos para a pessoa com mais de 65 anos. Além disso, $58,82 \%$ das idosas e $66,66 \%$ dos idosos afirmaram que, na ausência de problemas físicos graves, mulheres e homens podem manter o interesse e atividades sexuais até depois de 80 ou 90 anos de idade.

Em contrapartida, quando se pensa na atividade sexual como uma necessidade, o percentual de mulheres $(56,86 \%)$ que discordam é maior que o dos homens $(41,67 \%)$.

Apesar dos benefícios observados em relação às práticas sexuais, $42,15 \%$ das mulheres admitiram que fosse vergonhoso demonstrar interesse por sexo, ao contrário dos homens, que perfizeram $20,83 \%$ de concordância total ou parcial, o que traz a necessidade de se discutir questões relacionadas a gênero.

Em relação à afirmação de que o interesse sexual desaparece, inevitavelmente, após os 65 anos, $73,53 \%$ das mulheres concordaram total ou parcialmente e, na mesma direção, os homens, com 72,92\%.
Percebe-se, assim, que a maioria da população pesquisada compreende a vida sexual como um componente do estilo de vida daqueles que se mantêm saudáveis, mas também percebe que, de fato, não é uma realidade da maioria. Tanto assim que para $56,86 \%$ das entrevistadas e $41,67 \%$ dos entrevistados a sexualidade não é uma necessidade que se faz presente durante a vida toda.

Ao serem perguntados se a maioria das mulheres com mais de 65 anos é fria com relação ao sexo, $74,51 \%$ das mulheres e $62,50 \%$ dos homens responderam que era verdade. Para $68,63 \%$ das idosas e $58,34 \%$ dos idosos, nas mulheres a perda de satisfação sexual é inevitável após a menopausa. Em relação aos homens, especificamente, $67,65 \%$ das idosas e $62,50 \%$ dos idosos acreditam que é possível tratar a impotência sexual.

Ao serem questionados se é provável que o término da atividade sexual em pessoas com mais de 65 anos se deva prioritariamente a fatores sociais e psicológicos em vez de fatores biológicos e físicos, $58,82 \%$ das mulheres e $60,42 \%$ dos homens responderam ser verdade. O percentual, entretanto, é maior quando a pergunta refere-se ao receio da impotência, uma vez que para $77,45 \%$ das idosas e $72,92 \%$ dos idosos o medo de não ser capaz de realizar o ato sexual pode acarretar na incapacidade de um bom desempenho sexual em homens com mais de 65 anos. De acordo com $53,92 \%$ das entrevistadas e $62,50 \%$ dos entrevistados, medicamentos podem alterar o desejo sexual de uma pessoa.

A masturbação, outro tema investigado, apresenta-se como um assunto pouco conhecido e que vem acompanhado também de alguns tabus. Enquanto respondiam à escala, algumas mulheres faziam comentários tais como: "masturbação é coisa para homem" e "homem que faz isso", o que mostra que é algo proibido e/ou sequer imaginado para mulheres. Para $45,09 \%$ das participantes e $31,25 \%$ dos participantes, a masturbação em excesso pode causar o aparecimento de confusão mental e de demência em pessoas com mais de 65 anos. Além disso, $45,10 \%$ das mulheres e $43,75 \%$ dos homens alegaram não saberem se a masturbação traz benefícios para a respostas sexuais. Nessa mesma tendência, $45,09 \%$ das mulheres e $27,08 \%$ dos homens responderam discordar totalmente da ideia de que a masturbação é uma atividade sexual aceitável para homens com mais de 65 anos. 
Ao falarmos sobre a possibilidade de mulheres e homens com mais de 65 anos precisarem de parceiros mais jovens para se sentirem estimulados, $65,69 \%$ das idosas e $62,50 \%$ dos idosos consideraram uma inverdade. Nos comentários, ao longo da entrevista, afirmaram que "... se arrumar uma pessoa mais nova, vai levar gaia [traição], aí não adianta”. Portanto, é preciso considerar a hipótese de que esse percentual se deva ao receio de uma desilusão, e não propriamente a uma consideração de que não seja estimulante. Conforme Minayo, Meneghel e Cavalcante (2012), a traição é considerada um sofrimento para homens e mulheres. Para as autoras, alguns idosos do sexo masculino chegam a cometer suicídio diante de uma traição e existe um medo de se relacionarem com mulheres mais jovens por não apresentarem o mesmo potencial sexual que tinham quando jovens.

Os participantes desta pesquisa alegaram discordar totalmente $(50 \%$ das mulheres e $72,92 \%$ dos homens) da ideia de que é vergonhoso para uma pessoa com mais de 65 anos mostrar interesse por sexo. Apesar disso, é preciso retomar os dados já apresentados que apontam que a maioria considera que as mulheres se tornam frias sexualmente e que a satisfação sexual diminui após a menopausa. Assim, pode não causar necessariamente vergonha, mas parece que na opinião deles não é uma prática frequente.

Sobre a vivência sexual em instituições de longa permanência, $53,92 \%$ das idosas e $70,84 \%$ dos idosos discordaram totalmente da ideia de que casas de repouso não têm obrigação de garantir privacidade para seus moradores que desejem ficar a sós com seus companheiros.

Apontados os principais resultados advindos da opinião dos participantes, a seguir será discutida essa realidade encontrada.

\section{Discussão}

O convite para a participação da pesquisa nas ESF se deu sem havermos mencionado identidade de gênero e foi considerado o sexo declarado de cada participante, como já mencionado. A participação majoritária de mulheres pode ter relação com os dados encontrados na literatura sobre as diferenças de comportamento entre homens e mulheres quanto ao cuidado com a saúde. Keijzer (2016) discute sobre a forma como a masculinidade hegemônica construída interfere nos cuidados e nos enfrentamentos necessários para o cuidado. $\mathrm{O}$ autor levanta, entre outras dificuldades, o alcoolismo e a violência. Mesmo que não seja objetivo inicial da presente pesquisa, as questões de gênero estiveram presentes.

Sinković e Towler (2018), em revisão sistematizada, encontraram que os mitos e tendências às diferenças de gênero foram preponderantes em culturas não ocidentais. Comparações sistematizadas precisam ser realizadas para ampliar a compreensão desta semelhança encontrada entre idosas e idosos do agreste pernambucano e culturas de hábitos diferentes. Uma similaridade pode ser aventada quanto à vivência do patriarcado nas mais diversas culturas.

De Paula (2018) discute a relação entre patriarcado e direitos humanos apontando que, apesar de esses direitos serem considerados universais, na prática, a mulher tem sido historicamente subjugada. Assim, não é incoerente que as mulheres da amostra aqui investigada demonstrem uma tendência a relegar ao segundo plano uma dimensão que pode gerar autoconhecimento, prazer e autonomia.

Ainda corroborando esse argumento e retomando os dados de Sinković e Towler (2018), nossos dados confirmam o mito de que o desejo sexual da mulher diminui após a menopausa, uma vez que este percentual foi alto para os homens $(62,5 \%)$ e ainda maior para as mulheres $(74,51 \%)$.

Em relação ao interesse na prática sexual, mais da metade de nossa amostra considera que o interesse, inevitavelmente, desaparece. Algumas variáveis que precisam ser consideradas são a cultura local, a escolaridade e a baixa renda per capita do município, que podem influenciar no conhecimento e atitudes dos entrevistados. Portanto, se há relação entre promoção de saúde e manutenção do interesse sexual, tal como Štulhofer et al. (2018) apontam, há justificativas sedimentadas de que é necessário investir em políticas públicas que cuidem da sexualidade e da saúde integral das pessoas em processo de envelhecimento. Machado (2014) destaca que existem mudanças físicas com o passar dos anos, mas o prazer sexual pode ser mantido e seus benefícios são tanto físicos quanto psicológicos.

Nowakowski e Sumerau (2019), em um estudo de revisão de literatura, identificaram investigações que apontaram uma tendência para o fato de que o envelhecimento afeta o significado e a função das sexualidades, mas ainda assim a vivência dessa dimensão permanece importante e significativa em idades posteriores. Os dados da presente pesquisa 
caminham nessa mesma direção. As autoras também mencionam a importância de se investigar sobre o que exatamente é considerado sexual na vida de uma mulher. O sexo oral, por exemplo, foi considerado um comportamento sexual definidor para os parceiros e estava significativamente relacionado às percepções da qualidade sexual, apesar de variar conforme sexos, gêneros, idades e diferentes tipos de relacionamento. Apesar disso, nesta revisão, ao contrário, a maioria dos estudos encontrados focou na disfunção sexual masculina como predominantemente relacionada à ereção. Quando se tratava de mulheres, o tema era esterilização. Observa-se, mais uma vez, a necessidade de se ampliar as investigações quanto às práticas sexuais no envelhecimento.

O pressuposto de que a prática sexual é dificultada com o tempo pode ter uma conotação mais forte sobre as mulheres, mas também está presente nos homens. Alencar (2013) coloca que uma das crenças sobre a sexualidade na terceira idade é que os idosos se tornam assexuados, por se acreditar que o desejo sexual é cessado devido às mudanças fisiológicas, principalmente quando a mulher atinge a menopausa. A literatura corrobora o resultado, visto que $o$ uso de medicação e a presença de doenças crônicas podem interferir na resposta sexual dos indivíduos, aumentando ou diminuindo a libido (Carvalho \& Sardinha, 2017). Os autores destacam a revolução na vida sexual inicialmente de idosos do sexo masculino, que sofreu influência dos avanços farmacológicos, como o surgimento de medicamentos dedicados à melhora na impotência sexual, assim como relatam que pesquisas e produtos estão sendo feitos para o público feminino da terceira idade.

Para Rozendo e Alves (2015), a sexualidade para idosos pode estar vinculada ao carinho, à compreensão, às carícias e ao afeto. Para muitas pessoas a palavra sexualidade faz referência somente ao ato sexual, pois não percebem as diversas formas de vivenciar a sexualidade e de obter prazer sexual. A masturbação, como se viu nos dados aqui apresentados, é um tabu para a maior parte dos entrevistados. Outros autores encontraram situação similar. Hernández Carrasco et al. (2019), em estudo com 138 idosos de área urbana, identificaram que tanto para homens $(15,9 \%)$ como para mulheres (19,6\%), a penetração vaginal é a prática sexual mais comum. A masturbação apareceu apenas em 9,4\% - o estudo não diferenciou sexo ao fornecer esse dado -, o que coincide com a realidade do município da presente pesquisa. A cultura reforça a masturbação como uma prática masculina e para muitas mulheres ela é um tabu, dificultando que conheçam o próprio corpo (Carvalho \& Sardinha, 2017). Isso pode ter levado a maioria dos pesquisados a considerá-la como algo negativo e/ou prejudicial.

Levando em consideração que a sexualidade não se limita ao ato sexual, mas à forma como cada pessoa lida com sua imagem e seu corpo, e considerando ainda que outras respostas indicam que a maioria apresenta limitações nas atitudes e conhecimentos sobre sexualidade, podemos supor que a sexualidade é relegada a um segundo plano ou até desconsiderada (Alencar, 2013; Denney \& Quadagno, 1992; Viana, 2008).

Problemas de saúde podem afetar o desempenho sexual, como: artrites, diabetes, fadiga, medo de infarto, depressão, efeitos colaterais de fármacos, uso de álcool e outras drogas (Ministério da Saúde, 2006). Destarte, a frequência e a intensidade da atividade sexual mudam ao longo da vida, mas não se pode naturalizar a incapacidade de desfrutar de forma prazerosa das relações sexuais na terceira idade (Alencar, 2013). Os dados do presente estudo mostram que no município pesquisado a forma como o envelhecimento é compreendido pelos idosos coloca a mulher em uma situação de ter menor frequência de vivências sexuais, além da menor possibilidade de tê-las de formas satisfatórias. Isso é reflexo de uma sociedade de base patriarcal que apregoou a liberdade sexual para os homens e a proibiu para as mulheres. As respostas relacionadas a atitudes machistas compareceram tanto nos homens quanto nas mulheres. Ao longo da história, foram impostos quais comportamentos deveriam ser adotados por homens e quais seriam próprios das mulheres, diferenciação que é conceituada como papéis de gênero (J. Butler, 1998). As delimitações dos papéis sociais do feminino e do masculino interferem nos diversos âmbitos do sujeito, como sua sexualidade.

Precisamos considerar também a pluralidade das histórias de vida. Portanto, não é possível adotarmos conceitos únicos e deterministas. Lima, Espelt, Lima e Bosque-Prous (2018), em pesquisa transcultural envolvendo 17 países, encontraram que, em relação à limitação de atividades sexuais, as médias das mulheres foram significativamente mais altas $(\mathrm{g} 10=\mathrm{b} 1 \mathrm{j}=0,36$, $\mathrm{p}<0,001$ ), fenômeno acentuado no caso de ocorrência de desigualdades sociais de gênero.

$\mathrm{Na}$ amostra desta pesquisa, observou-se que as mulheres, mais que os homens, consideraram o sexo 
como perigoso para a saúde, afirmaram que a prática sexual não traz benefícios para a saúde, que a prática sexual não é necessária ao longo de toda a vida, que as mulheres com mais de 65 anos são frias sexualmente, que masturbação em excesso pode ocasionar demência ou confusão mental para homens e mulheres, além de perceberem como vergonhoso manter o interesse sexual com o passar dos anos. A relação entre sexualidade e casamento é decorrente de normas culturais e religiosas, conforme discutem Foucault (1988) e Viana (2008). Zanello, Silva e Henderson (2015) realizaram entrevistas com 18 moradores de uma instituição de longa permanência e discutiram como o sofrimento psíquico de pessoas institucionalizadas precisa considerar as relações de gênero. Apesar de ser uma pesquisa qualitativa, os dados apontam para direções semelhantes aos aqui encontrados, ou seja, as mulheres não mencionaram espontaneamente as práticas sexuais, enquanto aos homens incomodava a impotência.

Os participantes desta pesquisa demonstraram uma tendência a perceber o intercurso sexual como menos necessário e/ou menos possível no envelhecimento, posição predominante no caso das mulheres. Nesse aspecto nossos dados são similares à pesquisa de Sinković e Towler (2018).

De acordo com Debert e Brigueiro (2012), não é possível pensar em envelhecimento ativo sem considerar a sexualidade, tida como fundamental para a promoção de saúde das pessoas longevas. Eles defendem a inclusão da velhice no curso de vida sexual, assim como a necessidade de se considerar a ampliação das zonas erógenas para além dos órgãos genitais. Os autores apontam para a necessidade de se discutir o corpo erótico no envelhecimento não só relacionando à manutenção da saúde. Eles pensam também em um corpo passível de ser atraente, sensual, enfim, belo, criticando o entendimento das políticas públicas de saúde que associam a prática sexual a partir de uma ótica de respeitabilidade que a dissocia do hedonismo afrodisíaco. Na amostra aqui pesquisada, há ainda tantos mitos e tabus que a opinião dos idosos - incluindo as falas espontâneas ao longo da entrevista - passa pelos sentidos de "se ainda funcionar", "ainda ser possível", como se a atividade sexual fosse uma exceção; e eles sequer admitem a possibilidade de prazer. Considerando os dados das mulheres, então, tudo indica que a libido nunca pôde ser discutida e agora há apenas uma continuidade da mesma lógica que enquadra o sexo como função meramente reprodutiva.

Nowakowski e Sumerau (2019) nos falam que o envelhecimento, ao afetar o significado e a função das sexualidades, pode trazer novas representações que vão interferir na vida sexual dos idosos. No caso das mulheres, as autoras destacam a relevância de se investigar o que significa uma vida sexual ativa e satisfatória para elas. O tema sexualidade é repleto de crenças limitadoras que trazem diferenças importantes para homens e mulheres. Os dados aqui encontrados, nesta amostra de um município do agreste pernambucano, também demonstram perpetuação de tabus e, especificamente nesta população, o prazer não pode sequer ser cogitado. Em linhas gerais, a saúde sexual de mulheres acima de 60 anos precisa ser amplamente considerada em pesquisas, com vistas a oferecer maior profundidade nos entendimentos, tanto na clínica quanto na academia.

O estudo do envelhecimento na perspectiva de promoção de saúde pode ampliar a visão sobre os cuidados para outras etapas do curso de vida. Para que haja envelhecimentos diferenciados, é preciso investir igualmente em vidas diferentes. Essa perspectiva traz também a necessidade de se investir na saúde sexual de mulheres de qualquer idade e nas sexualidades dos homens também. Nesse sentido, as realidades e sentidos encontrados junto aos idosos podem direcionar políticas públicas para outras faixas etárias.

Leandro-França e Murta (2014), além de colocarem a importância de estudos na área da promoção de saúde no envelhecimento, retratam a realidade de que ainda há uma preponderância na literatura de estudos voltados à infância e à juventude. Defendemos, portanto, não apenas a necessidade de se ampliarem as investigações para promoção de saúde no envelhecimento pelos benefícios a essa população, mas também devido às vantagens que esse conhecimento pode trazer para todo o curso de vida. Para isso, os profissionais de saúde podem ajudar a desmistificar o assunto sexualidade na terceira idade.

\section{Considerações finais}

Pensamentos cristalizados sobre o que é ser mulher e ser homem impõem comportamentos sexuais que limitam as expressões e desejos de homens e mulheres. Intervenções com mulheres para desmistificar as limitações impostas pelas questões de gênero é uma necessidade, para possibilitar que a autonomia 
feminina aconteça e contribua na (re)construção de uma sexualidade que traga bem-estar e realização.

O imaginário acerca do que é envelhecer e de qual podem ser os espaços dos idosos na sociedade parece interferir diretamente no intercurso sexual. Uma população com menos acesso à saúde e, principalmente, à educação, tende a construir mitos acerca das vivências sexuais, que a deixam à margem dos benefícios e conhecimentos produzidos pela ciência.

Ao conhecer os estereótipos apresentados fica evidente que, em especial as idosas, não tiveram contato com esse tema, com assuntos que não eram permitidos e/ou eram motivos de vergonha. Temas relacionados à sexualidade e ao sexo se mostraram mais permitidos aos homens, que são incentivados histórico e socialmente a falar e agir com maior liberdade no âmbito da sexualidade. Levar ao rompimento dessas crenças e construir novos significados, sentidos e comportamentos pode tornar os idosos mais ativos. Assim, existe a necessidade de que cada vez mais os profissionais da saúde e de outras áreas trabalhem com sexualidade na terceira idade, com a criação de ambientes para discussão sobre a temática, grupos, palestras, formações e pesquisas que contribuam com o aperfeiçoamento profissional e para a abertura do assunto na sociedade.

Todas as camadas da sociedade devem estar preparadas para proporcionar o bem-estar aos idosos, com destaque para as políticas públicas que devem apresentar estratégias para suprir suas necessidades.
O número de idosos aumenta a cada ano, motivo pelo qual surge a necessidade de intervir positivamente na forma como estão vivendo essa fase do desenvolvimento humano. Nossa população está envelhecendo e os idosos precisam ser vistos em seus amplos aspectos, entre eles, a sexualidade.

A sexualidade se faz presente independentemente da idade, pois o prazer sexual não acaba com o passar dos anos. Por isso, deve-se lembrar que existem meios alternativos para a satisfação sexual que vão além da penetração, como o uso de vibradores, sexo oral, massagens, toques e caricias, entre outros. Além disso, há formas de facilitar a relação sexual, como o uso de medicações, lubrificantes, terapias e acompanhamento médico. Para ter acesso a essas informações, é necessário que os profissionais e os idosos saibam a relevância da sexualidade para o bem-estar.

Destarte, existem mudanças nas respostas sexuais com o passar dos tempos, mas isso não significa que o idoso seja assexuado. A forma como o casal ou o indivíduo construiu suas relações amorosas e sexuais influencia a maneira como a sexualidade e/ou o ato sexual será vivenciado de forma conjunta ou individual na terceira idade.

Os resultados aqui obtidos apontam para a necessidade de se investir em programas de cuidado da sexualidade das pessoas durante o processo de envelhecimento, uma dimensão que precisa compor as ações de promoção de saúde nas esferas municipal, estadual e federal.

\section{Referências}

Alencar, D. L. (2013). Fatores associados ao exercício da sexualidade de pessoas idosas [Dissertação de mestrado, Universidade Federal de Pernambuco]. Attena: Repositório Digital da UFPE. http://www.repositorio.ufpe.br/ handle/123456789/11074

Alencar, D. L., Marques, A. P. O., Leal, M. C. C., \& Vieira, J. C. M. (2014). Fatores que interferem na sexualidade de idosos: Uma revisão integrativa. Ciência \& Saúde Coletiva, 19(8), 3533-3542. https://doi.org/ 10.1590/1413-81232014198.12092013

Butler, R., \& Lewis, M. (1985). Sexo e amor na terceira idade (I. de Carvalho Filho, Trad.; 2a ed.). Summus.

Butler, J. (1998). Fundamentos contingentes: O feminismo e a questão do pós-modernismo (P. M. Soares, Trad.). Cadernos Pagu, (11), 11-42. (Artigo original publicado em setembro de 1990)

Hernández Carrasco, M., Fuente Ballesteros, S. L., García Granja, N., Hidalgo Benito, A., García Álvarez, I., \& Cano Pazos, M. (2019). Características de la esfera sexual en pacientes adultos mayores. Semergen: Revista Española de Medicina de Família, (1), 37-43. https://dialnet.unirioja.es/servlet/articulo? codigo=6769680

Carvalho, A., \& Sardinha, A. (2017). Terapia cognitiva sexual: Uma proposta na psicoterapia sexual. Cognitiva.

Debert, G,. \& Brigeiro, M. (2012). Fronteiras de gênero e a sexualidade na velhice. Revista Brasileira de Ciências Sociais, 27(80), 37-54. https://doi.org/10.1590/S0102-69092012000300003

Denney, N. W., \& Quadagno, D. (1992). Human sexuality (2nd. ed.). Mosby Year Book. 
De Paula, D. O. (2018). Human rights and violence against women: Campo Algodonero case. Revista Estudos Feministas, 26(3). https://doi.org/10.1590/1806-9584-2018v26n358582

Esperón, J. M. T. (2017). Pesquisa quantitativa na ciência da enfermagem. Escola Anna Nery, 21(1). http:// www.scielo.br/pdf/ean/v21n1/1414-8145-ean-21-01-e20170027.pdf

Figueiredo, A. H. (Org.). (2016). Brasil: Uma visão geográfica e ambiental no início do século XXI. Coordenação de Geografia do IBGE. https://biblioteca.ibge.gov.br/visualizacao/livros/liv97884.pdf

Foucault, M. (1988). História da sexualidade I: A vontade de saber. Edições Graal.

Hogan, R. (1985). Human sexuality: A nursing perspective (2a ed.). Appleton Century Crofts.

Instituto Brasileiro de Geografia Estatística. (2010). Sinopse do Censo Demográfico de 2010. https:/ / censo2010.ibge. gov.br/sinopse/

Instituto Brasileiro de Geografia e Estatística. (2018). Projeções e estimativas da população do Brasil e das Unidades da Federação.

Jordão Netto, A. (1997). Gerontologia básica. Lemos.

Keijzer, B. (2016). "Sé que debo parar, pero no sé cómo": Abordajes teóricos en torno a los hombres, la salud y el cambio. Sexualidad, Salud y Sociedad, (22), 278-300. https://doi.org/10.1590/1984-6487.sess.2016.22.12.a

Leandro-França, C., \& Murta, M. G. (2014). Prevenção e promoção da saúde mental no envelhecimento: conceitos e intervenções. Psicologia: Ciência e Profissão, 34(2), 318-329. https://doi.org/10.1590/1982-3703001152013

Lima, A. L. B., Espelt, A., Lima, K. C., \& Bosque-Prous, M. (2018). Activity limitation in elderly people in the European context of gender inequality: A multilevel approach. Ciência \& Saúde Coletiva, 23(9), 2991-3000. https://doi.org/ $10.1590 / 1413-81232018239.20662016$

López Sánchez, F., \& Fuertes, A. (1989). Para compreender la sexualidad.Verbo Divino.

Machado, D. J. C (2014). Quem foi que disse que na terceira idade não se faz sexo?. Fragmentos de Cultura, 24(spe.), 11-14.

Minayo, M. C. S., Meneghel, S. N., \& Cavalcante, F. G. (2012). Suicídio de homens idosos no Brasil. Ciência \& Saúde Coletiva, 17(10), 2665-2674. https://doi.org/10.1590/S1413-81232012001000016

Ministério da Saúde. (2006). Envelhecimento e saúde da pessoa idosa. https://bvsms.saude.gov.br/bvs/publicacoes/evelhecimento_saude_pessoa_idosa.pdf

Nowakowski, A. C. H., \& Sumerau, J. E. (2019). Women's sexual health in later life: Gaps and opportunities in research and practice. Women's Health, 15. https://doi.org/10.1177/1745506519878092

Papaléo Netto, M. (2011). O estudo da velhice: Histórico, definição do campo e termos básicos. In E. V. Freitas, L. Py, F. A. X. Cançado, J. Doll \& M. L. Gorzoni, (Orgs.), Tratado de geriatria e gerontologia (3a ed., pp. 62-75). Guanabara Koogan.

Rozendo, A. S., \& Alves, J. M. (2015). Sexualidade na terceira idade: Tabus e realidade. Revista Kairós Gerontologia, 18(3), 95-107.

Sinković, M., \& Towler, L. (2018). Sexual aging: A systematic review of qualitative research on the sexuality and sexual health of older adults. Qualitative Health Research, 29(9), 1239-1254. https://doi.org/10.1177/ 1049732318819834

Štulhofer, A., Hinchliff, S., Jurin, T., Hald, G. M., \& Træen, B. (2016). Successful aging and changes in sexual interest and enjoyment among older European men and women. The Journal of Sexual Medicine, 15(10), 1393-1402. https:// doi.org/10.1016/j.jsxm.2018.08.011

Viana, H. B. (2008). Adaptação e validação da escala ASKAS: Aging Sexual Knowledgeand Atitudes Scale em idosos brasileiros [Tese de doutorado, Universidade Estadual de Campinas].

Viana, H. B., Guirardello, E. B., Madruga, V. A. (2010). Tradução e adaptação cultural da escala ASKAS: Aging Sexual Knowledge and Attitudes Scale em idosos brasileiros. Texto e Contexto: Enfermagem, 19(2), 238-245. https://doi.org/10.1590/S0104-07072010000200004

Zanello, V., Silva, L. C., \& Henderson, G. (2015). Saúde mental, gênero e velhice na instituição geriátrica. Psicologia: Teoria e Pesquisa, 31(4), 543-550. https://doi.org/10.1590/0102-37722015042444543550 


\section{Iara de França Santos}

Psicóloga Comportamental pela Universidade Federal de Alagoas (Ufal), Maceió - AL, Brasil. Pós-graduada em Saúde Pública pela Faculdade de Ensino Regional Alternativa (Fera), Arapiraca-AL, Brasil. Mestre pela Universidade de Pernambuco (UPE), Garanhuns - PE, Brasil. Foco de estudo: Sexualidade Humana.

E-mail: iarapsiufal@gmail.com

(D) https://orcid.org/0000-0002-8751-2839

Endereço para envio de correspondência:

Rua Tancredo de Almeida Neves, 51,Bairro Centro. CEP:57275-000. São Sebastião - AL. Brasil.

Recebido 13/03/2020

Aceito 03/05/2021

Received 03/13/2020

Approved 05/03/2021

Recibido 13/03/2020

Aceptado 03/05/2021

Como citar: Santos, I. de F. (2022). Atitudes e conhecimentos de idosos sobre intercurso sexual no envelhecimento. Psicologia: Ciência e Profissão, 42, 1-11. https://doi.org/10.1590/1982-3703003235106

How to cite: Santos, I. de F. (2022). Attitudes and knowledge of older adults about sexual intercourse in aging. Psicologia: Ciência e Profissão, 42, 1-11. https://doi.org/10.1590/1982-3703003235106

Cómo citar: Santos, I. de F. (2022). Actitudes y conocimientos de ancianos sobre relaciones sexuales en el envejecimiento. Psicologia: Ciência e Profissão, 42, 1-11. https://doi.org/10.1590/1982-3703003235106 